\title{
Über Wonne und Würde des Alters
}

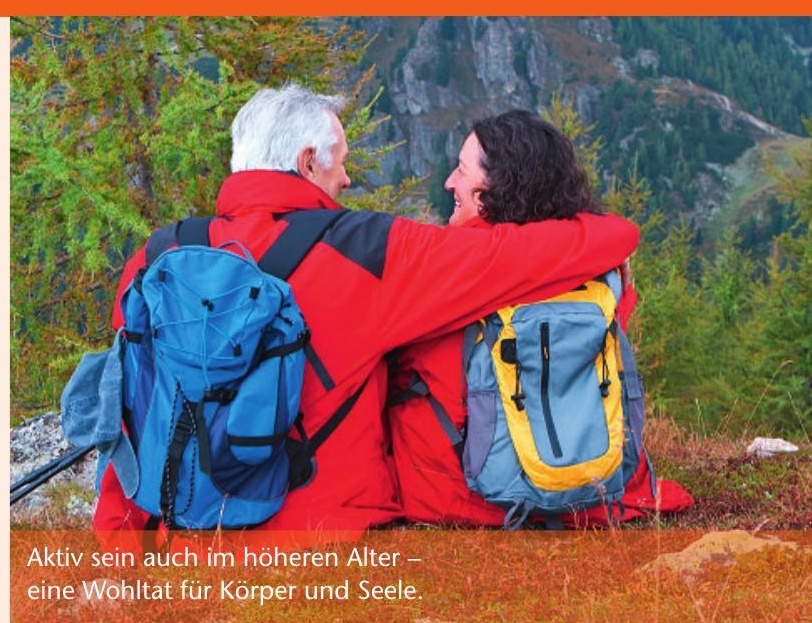

Peter Marko

1 Taverna E. Die Würde des Alters. Schweiz Ärztezeitung. 2009;90(35):1360.

Korrespondenz: Peter Marko Heinestrasse 26 CH-9008 St. Gallen

peter.j.marko@hin.ch
Erlauben Sie mir, lieber Herr Kollege Taverna, vielleicht nicht nur in meinem Namen, sondern auch anderer Greise, Grufties, Senioren (ich stehe im 73. Lebensjahr, also bin ich schon älter als Frau B. aus Brechts Kalendergeschichte, die Sie am Anfang Ihres Artikels [1] erwähnen) zu Ihren Überlegungen und Ausführungen Stellung zu nehmen. Ich bin Ihnen ausgesprochen dankbar, dass Sie mir Anlass zum Sammeln und Formulieren meiner Empfindungen und Gedanken zu diesem Thema gegeben haben.

Ja, ich crawle und schwimme auf dem Rücken, fahre Ski, wandere, übe fast jeden Morgen und achte auf meine Ernährung eifrig, fanatisch, fast zwanghaft. Ich arbeite auch noch. Warum? Ich tue das alles zwar viel langsamer, schwächer, aber mit grosser Freude, Befriedigung, Genugtuung und Dankbarkeit, dass ich es immer noch tun kann, dass ich es schaffe, ähnlich dem Kind, das sich freut, dass es schon greifen, laufen, sprechen kann. Wissen Sie, wie schön es war, der Enkelin das Skifahren und Schwimmen beizubringen, sie dabei zu begleiten, ihre Freude und ihren Stolz zu beobachten, mit ihr zu wandern? Kann man mir es übelnehmen, dass ich gerne, wenn es mir vergönnt sein wird, auch mit den weiteren Grosskindern all dies erleben möchte, und mich bemühe, das Meinige zur Erfüllung dieses Wunsches beizutragen?

Die Wanderungen sind ein besonderes Erlebnis. Während der vollen Berufsjahre haben wir wenig Zeit dafür gehabt. Jetzt entdecken wir neue Wege oder schreiten die alten ohne Eile in gemächlichem Tempo, weil es nicht mehr darum geht, wie schnell wir einen Weg schaffen, sondern es uns freut, dass wir es überhaupt noch können. Dabei vertiefen wir uns in und kauen an den frei schwebenden Gedanken und Erinnerungen. Es gibt für uns keine bessere Ablenkung von den täglichen Sorgen, Problemen, keine bessere Erholung.

Nicht nur von anderen Altersgenossen, denen es leider nicht vergönnt ist, so aktiv zu bleiben, weiss ich, dass es nicht selbstverständlich ist. Auch ich erlebte Zeiten, als die Nerven in der Lenden- und dann Halswirbelsäule eingeklemmt waren und meine Bewegungsfreiheit stark beschränkt war. Seither lernte ich besser Crawlen und Rückenschwimmen, weil das Brustschwimmen immer noch zu Beschwerden führt.
Aber wissen Sie, lieber Herr Kollege Taverna, wie schön es ist, in dem Weiher oberhalb unserer Stadt unter den über dem Wasser hängenden Ästen der Uferbäume auf dem Rücken neben den Enten zu schwimmen, und am frühen Morgen die oben zwitschernden Vögel dabei zu beobachten? Jahrelang litt ich auch unter den trotz mehrerer Operationen schmerzhaften Leistenbrüchen. Jetzt, nach der laparoskopischen Operation mit Netz kann ich wieder unbeschränkt Lasten heben, mich bewegen - ich bin jünger, leistungsfähiger geworden!

Wir achten sehr auf die Ernährung. Auch dieser Bereich ist im Alter empfindlicher und weniger belastbar. Man sollte sich dem anpassen. Wir merken, was uns nicht gut tut, korrigieren es und bemühen uns, das Gewicht zu halten, weil wir mit jedem Kilo spüren, wie wir uns schwerfälliger bewegen.

Ich arbeite mit der Hilfe meiner Frau noch. Anders als früher. Wir haben keine Apparate, aber Zeit für die Patienten. Ich treibe einen psychosomatischen, bioenergetischen Zugang zu ihren Problemen, mit gezieltem Ausgleich von Vitaminen, Mineralien und Spurenelementen, wenn sie fehlen, und einer gesunden, einfachen, abwechslungsreichen Ernährung als unabdingbare Voraussetzungen für Heilung und Gesundheit. Ich versuche, den Patienten auch meine Einstellung zur Bewegung und zur Lösung der Probleme zu vermitteln. Davon leben könnten wir aber sicher nicht.

Vor kurzem habe ich ein neues Auto gekauft. (Das kleinste Modell der Firma, für unsere Bedürfnisse reicht es vollständig, da wir dank dem GA der SBB längere Strecken mit der Bahn fahren.) Dabei wurde mir bewusst, dass es der letzte Wagen meines Lebens ist. Unterschwellig ist diese Bedrohung, Mahnung, dieses Gefühl eigentlich ständig da. Weiss ich, ob ich noch ...? Selbstverständlich werden sich meine Fähigkeiten und Möglichkeiten weiter verringern. Wenn ich einmal auf diese Tätigkeiten ganz verzichten müsste und noch geistig fähig wäre, würde ich mich freuen, wenn meine Frau sie dann auch für mich geniessen und mir ihre Erlebnisse mitteilen würde.

Sie befürchten, dass wir Senioren das alles (nur) unter Druck tun, weil uns sonst vorgeworfen wird, unnötig Kosten für unsere Behandlungen zu verursachen. Diesem «sozialen Druck» möchten Sie uns 
nicht aussetzen. Wie ich geschildert habe, bewege ich mich hauptsächlich für mich und meine Nächsten. Aber ich bin froh, dass ich dabei auch die Allgemeinheit, die Mitmenschen möglichst nicht unnötig belaste, so wie ich im Treppenhaus, auf der Strasse und in der Natur meine Abfälle nicht hinterlasse, den gemeinsamen Besitz nicht schädige. Wie wir aus neueren Untersuchungen wissen, beeinflusst unser Verhalten die Verbreitung vieler guter und schlechter Gewohnheiten auch im gesundheitlichen Bereich. Auch Dank dem sozialen Druck und der entsprechenden Aufklärung erreichten wir unseren hohen hygienischen, gesundheitlichen und zivilisatorischen $\mathrm{Zu}-$ stand. Kann man ihn bei Bemühungen um Änderungen, Fortschritt überhaupt irgendwie «weglassen»? Dasselbe betrifft die Bedeutung der finanziellen Anreize. Im Gesundheitsbereich denken wir dabei vorwiegend an «Strafen», auch wenn Belohnung, z. B. bei einer (dauerhaften) Gewichtsabnahme, wirksamer ist als andere Massnahmen. Die Kraft der (schlecht gestalteten) finanziellen Anreize, der Boni, wurde uns vor kurzem demonstriert.

\section{Ich arbeite mit der Hilfe meiner Frau noch. Anders als früher. Wir haben keine Apparate, aber Zeit für die Patienten}

Sie zweifeln auch, ob wir die üblichen hohen Kosten der letzten sechs Monate des Lebens sparen, wenn wir es auf gesunde Weise verlängern. Während meiner jahrzehntelangen Praxis habe ich oft mit alten Menschen zu tun gehabt, die sich nicht um den Tod sorgten, sondern um das Leiden dabei, um die Abhängigkeit, um das «zur Last fallen», um den "Anschluss an Schläuche und Maschinen». Ich habe ihnen, meine Mutter eingeschlossen, immer wieder gesagt, je länger sie leben, desto schneller und leichter kommt der Tod. Es ist sowohl körperlich wie psychisch begründet. Ein Mensch in den Siebzigern, noch nach dem Leben hungrig, nimmt eher in Kauf, dass von der All- gemeinheit tausende Franken für die Verlängerung seines Lebens um ein paar Monate ausgegeben werden, als ein Neunzigjähriger. Aber ich habe auch über achtzigjährige Patienten gehabt, die sagten, das Jahr nach der Operation des Magenkrebses bis zum Tod sei trotz des Leidens sehr schön und wertvoll für sie und ihre Angehörigen gewesen. Ja, mit den Jahren ändert sich die Einstellung zum Leben und zum Tod.

Ich weiss, je länger man lebt, desto länger bezieht man die Rente und verursacht auf dieser Seite Kosten. Ich denke, mit der Zeit werden wir immer mehr schätzen, wenn wir den Möglichkeiten und Fähigkeiten entsprechend länger arbeiten können. Zum Leben, zum Menschsein gehört auch, nützlich zu sein, etwas (nicht nur sich) zu bewegen, zu wirken, ändern, einen Lebenssinn zu haben. Solche kulturellen Änderungen geschehen manchmal relativ schnell. Bei der jungen Generation zum Beispiel steigt die Geburtenrate, weil sie begriffen hat, dass zu einem erfüllten Leben auch Kinder gehören.

Durch neue Erkenntnisse und Umstände wurden wir aus dem Paradies der Sorglosigkeit auf die Erde der Verantwortung vertrieben. Es wird uns nicht mehr vergeben, weil wir inzwischen wissen - in meiner Kindheit war es noch nicht so - dass Rauchen, falsche Ernährung, wenig Bewegung und Umweltverschmutzung unsere Gesundheit wesentlich beeinträchtigen und das Leben verkürzen. Die kurativen Massnahmen dagegen sind immer teurer, nebenwirkungsreicher und ihre Kosten kann kaum jemand selbst tragen. Das war vor nicht allzu langer Zeit auch nicht so. Deswegen ist die Vorbeugung so wichtig. Zugegeben, sie ist für viele Menschn naturgemäss nicht leicht, aber auch nicht unmöglich. Wir sollen ihnen dabei wirksam helfen, aber sie nicht bestrafen. Neuerlich geben (noch nicht in der Schweiz) die Rentenversicherungen den Rauchern und Übergewichtigen Boni (gibt es eine bessere Warnung vor diesen Gefahren?). Wieso sollten Krankenkassen sie den Nichtrauchern und den Normgewichtigen nicht auch gewähren?

Ich glaube, aus verschiedenen guten Gründen wird die Schweiz bei der neuen Einstellung zu Chancen, Aufgaben, Pflichten, Genüssen und Würde des Alters weiterhin eine Vorreiterrolle spielen. 